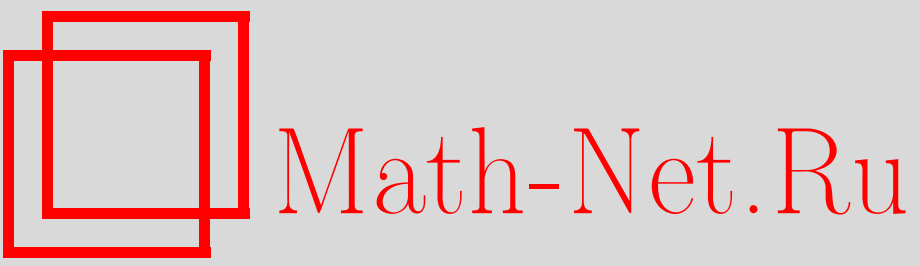

И. В. Баскаков, Когомологии $K$-степеней пространств и комбинаторика симплициальных разбиений, УМH, 2002, том 57, выпуск 5, 147-148

DOI: https://doi.org/10.4213/rm558

Использование Общероссийского математического портала Math-Net.Ru подразумевает, что вы прочитали и согласны с пользовательским соглашением

http://www.mathnet.ru/rus/agreement

Параметры загрузки:

IP : 34.229 .108 .108

26 апреля 2023 г., 05:05:59 


\title{
КОГОМОЛОГИИ К-СТЕПЕНЕЙ ПРОСТРАНСТВ И КОМБИНАТОРИКА СИМПЛИЦИАЛЬНЫХ РАЗБИЕНИЙ
}

\author{
И. В. БАСКАКОВ
}

Пусть $K$ - симплициальный комплекс, $V=\left\{v_{1}, \ldots, v_{m}\right\}$ - множество его вершин. Определим $K$-степень пары топологических пространств $(X, W)$ следующей формулой:

$$
\mathscr{Z}_{K}(X, W)=\bigcup_{\sigma \in K} X^{\sigma} \times W^{V \backslash \sigma} \subset X^{V},
$$

где $X^{\sigma} \times W^{V \backslash \sigma}=\left\{\left(x_{1}, \ldots, x_{m}\right) \mid x_{i} \in X\right.$, если $i \in \sigma$, иначе $\left.x_{i} \in W\right\}$ (см. [1]).

В настоящей статье изучаются когомологии пространства $\mathscr{Z}_{K}(X, W)$ и описьвается их связь с комбинаторикой симплициального разбиения $K$.

Как легко видеть, конструкция пространства $\mathscr{Z}_{K}(X, W)$ функториальна относительно отображения пар $\left(X_{1}, W_{1}\right) \rightarrow\left(X_{2}, W_{2}\right)$. Кроме того, если $X$ - топологический коммутативньй моноид, $W$ - подмоноид, то $\mathscr{Z}_{K}(X, W)$ превращается в функтор на категории симплициальных комплексов следующим образом. Пусть $\varphi: K^{\prime} \rightarrow K^{\prime \prime}$ - симплициальное отображение комплекса $K^{\prime}$ в $K^{\prime \prime}, V^{\prime}=\left\{v_{1}^{\prime}, \ldots, v_{m^{\prime}}^{\prime}\right\}$ и $V^{\prime \prime}=\left\{v_{1}^{\prime \prime}, \ldots, v_{m^{\prime \prime}}^{\prime \prime}\right\}$ - их множества вершин, тогда индуцированное отображение $\varphi \mathscr{Z}$ задается по формуле:

$$
\varphi_{\mathscr{Z}}\left(\left(x_{1}, \ldots, x_{m^{\prime}}\right)\right)=\left(\prod_{\varphi\left(v_{j}^{\prime}\right)=v_{1}^{\prime \prime}} x_{j}, \ldots, \prod_{\varphi\left(v_{j}^{\prime}\right)=v_{m^{\prime \prime}}^{\prime \prime}} x_{j}\right) .
$$

Более того, для пары некоммутативных моноидов $(X, W)$ любое симплициальное отображение $K^{\prime} \rightarrow K^{\prime \prime}$, сохраняющее порядок вершин, индуцирует отображение пространств $\mathscr{Z}_{K}(X, W)$ по указанной выше формуле.

Единичньй диск $D^{2} \subset \mathbb{C}$ в поле комплексных чисел дает пару коммутативных моноидов $\left(D^{2}, \partial D^{2}\right)$. Пространство $\mathscr{Z}_{K}\left(D^{2}, \partial D^{2}\right)$ называется момент-угол-комплексом $\mathscr{Z}_{K}$ [1]. Впервые это пространство появилось в [2]. Детальное изучение его свойств дано в [1]. В [1] в кольце когомологий момент-угол-комплекса была введена биградуировка. Следующая теорема описывает структуру биградуированного колцц когомологий $\mathscr{Z}_{K}$ через когомологии полных подкомплексов в $K$.

Теорема 1. Имеет место функториальный изоморфизм:

$$
H^{-j, 2 i}\left(\mathscr{Z}_{K}\right)=\bigoplus_{\gamma \subset V,|\gamma|=i} \widetilde{H}^{i-j-1}\left(K_{\gamma}\right),
$$

где $K_{\gamma}=\{\eta \in K \mid \eta \subset \gamma\}-$ полный подкомплекс на вершинах из $\gamma$. Кроме того, если $\alpha \in \widetilde{H}^{i}\left(K_{\gamma_{1}}\right) u \chi \in \widetilde{H}^{j}\left(K_{\gamma_{2}}\right), m o$

$$
\alpha \smile \chi= \begin{cases}0, & \gamma_{1} \cap \gamma_{2} \neq \varnothing, \\ i^{*} \lambda^{*}(\alpha \otimes \chi), & \gamma_{1} \cap \gamma_{2}=\varnothing,\end{cases}
$$

где $\lambda: \widetilde{C}^{*}\left(K_{\gamma_{1}}\right) \otimes \widetilde{C}^{*}\left(K_{\gamma_{2}}\right) \rightarrow \widetilde{C}^{*+1}\left(K_{\gamma_{1}} * K_{\gamma_{2}}\right)-$ изоморфизм, $i: K_{\gamma_{1}} \sqcup \gamma_{2} \rightarrow K_{\gamma_{1}} * K_{\gamma_{2}}-$ вложение, а $K_{\gamma_{1}} * K_{\gamma_{2}}-$ джойн $K_{\gamma_{1}}$ и $K_{\gamma_{2}}$.

Рассмотрим теперь кольцо Стенли-Райснера $\mathbb{k}(K)[3]$, т.е. фактор-кольцо $\mathbb{k}\left[v_{1}, \ldots, v_{m}\right] / \mathscr{I}$, где $\mathscr{I}$ - идеал в кольце многочленов $\mathbb{k}\left[v_{1}, \ldots, v_{m}\right]$, порожденньй мономами $v_{i_{1}} \cdots v_{i_{k}}$ такими, что $\left\{v_{i_{1}}, \ldots, v_{i_{k}}\right\} \notin K$.

Согласно [1; теорема 4.2.2] имеет место изоморфизм биградуированных алгебр

$$
H^{*, *}\left(\mathscr{Z}_{K} ; \mathbb{k}\right) \cong \operatorname{Tor}_{\mathbb{k}\left[v_{1}, \ldots, v_{m}\right]}^{*, *}(\mathbb{k}(K), \mathbb{k}),
$$

где $\mathbb{k}$ - поле. Из этого результата и теоремы 1 получаем следующую теорему.

ТЕОРема 2. Для любого поля $\mathbb{k}$ имеет место функториальный изоморфизм алгебр

$$
\left.\bigoplus_{\gamma \subset V,|\gamma|=i} \widetilde{H}^{i-j-1}\left(K_{\gamma} ; \mathbb{k}\right) \cong \operatorname{Tor}_{\mathbb{k}\left[v_{1}, \ldots, v_{m}\right]}^{-j(k)}(K), \mathbb{k}\right)
$$


Аддитивный вариант этой теоремы дает известная теорема Хохстера [4].

Функториальость в теоремах 1 и 2 понимается в следующем смысле. Пусть $\varphi: K^{\prime} \rightarrow K^{\prime \prime}$ симплициальное отображение, тогда оно индуцирует гомоморфизм $\varphi_{\mathscr{Z}}^{*}$ биградуированных колец когомологий $\mathscr{Z}_{K}$, который следующим образом согласован с указанным в теореме 1 разложением $H^{-j, 2 i}\left(\mathscr{Z}_{K}\right)$. Пусть $\varphi_{\mathscr{Z}, \gamma^{\prime}, \gamma^{\prime \prime}}^{*}=\left.\pi_{\widetilde{H}^{*}\left(K_{\gamma^{\prime}}\right)} \circ \varphi_{\mathscr{Z}}^{*}\right|_{\widetilde{H}^{*}\left(K_{\gamma^{\prime \prime}}\right)}-$ проекция ограничения $\varphi \mathscr{Z}$ на когомологии полных подкомплексов, где $\pi_{\widetilde{H}^{*}\left(K_{\gamma^{\prime}}\right)}$ - проекция на прямое слагаемое. Тогда $\varphi_{\mathscr{Z}, \gamma^{\prime}, \gamma^{\prime \prime}}^{*}=\varphi_{\gamma^{\prime}, \gamma^{\prime \prime}}^{*}$, если $\varphi\left(\gamma^{\prime}\right)=\gamma^{\prime \prime}$ и $\left|\gamma^{\prime}\right|=\left|\gamma^{\prime \prime}\right|$, где $\varphi_{\gamma^{\prime}, \gamma^{\prime \prime}}: K_{\gamma^{\prime}} \rightarrow K_{\gamma^{\prime \prime}}$ индуцировано $\varphi$, и $\varphi_{\mathscr{Z}}, \gamma^{\prime}, \gamma^{\prime \prime}=0$ во всех остальных случаях. Наконец, отображение Тог-алгебр кольца Стенли-Райснера в правой части в теореме 2 индуцируется $\varphi_{\mathbb{k}}^{*}: \mathbb{k}\left[v_{1}^{\prime \prime}, \ldots, v_{m^{\prime \prime}}^{\prime \prime}\right] \rightarrow \mathbb{k}\left[v_{1}^{\prime}, \ldots, v_{m^{\prime}}^{\prime}\right]$ таким, что $\varphi_{\mathbb{K}}^{*}\left(v_{i}^{\prime \prime}\right)=\sum_{\varphi\left(v_{j}^{\prime}\right)=v_{i}^{\prime \prime}} v_{j}^{\prime}$.

Рассмотрим теперь точную последовательность клеточной пары $(X, W)$ над полем $\mathbb{k}$, пусть $\delta^{*}: H^{*}(W ; \mathbb{k}) \rightarrow H^{*+1}(X, W ; \mathbb{k})$ - кограничный оператор. Положим $A=\operatorname{Ker}\left(\delta^{*}\right)$, $\widehat{B}=\operatorname{Im}\left(\delta^{*}\right)$; выберем $B \subset H^{*}(W ; \mathbb{k})$ и $C \subset H^{*}(X, W ; \mathbb{k})$ так, чтобы $A \oplus B=H^{*}(W ; \mathbb{k})$ и $\widehat{B} \oplus C=H^{*}(X, W ; \mathbb{k})$. Следующий резултат описьвает аддитивную структуру колшца когомологий $\mathscr{Z}_{K}(X, W)$.

TeOpema 3.

$$
H^{*+1}\left(\mathscr{Z}_{K}(X, W) ; \mathbb{k}\right)=\bigoplus_{\sigma \in K} \bigoplus_{\substack{\beta \subset V \\ \beta \cap \sigma=\varnothing}} \widetilde{H}^{*}\left(\operatorname{link}_{K_{\sigma \cup \beta}} \sigma ; \mathbb{k}\right) \otimes C^{\sigma} \otimes B^{\beta} \otimes A^{V \backslash \sigma \sqcup \beta}
$$

Напомним, что $K$ является горенштейновым* [3] комплексом над $\mathbb{k}$, если когомологии $c$ коэффициентами в $\mathbb{k}$ линка любого симплекса из $K$ равны когомологиям сферы соответствующей размерности.

Из теоремы 3 для пары $\left(M^{n}, \partial M^{n}\right)$, где $M^{n}$ - многообразие, а $\partial M^{n}$ - его граница, получаем в качестве следствия

ПредЛОЖЕНИЕ 1. Если $K$ - горенштейнов ${ }^{*}$ комплекс над полем $\mathbb{k}$, то для $\mathscr{Z}_{K}\left(M^{n}\right.$, дм $\left.{ }^{n}\right)$ имеет место двойственность Пуанкаре над $\mathbb{k}$, которая переводит $\widetilde{H}^{*}\left(\operatorname{link}_{K_{\sigma \cup \beta}} \sigma ; \mathbb{k}\right) \otimes C^{\sigma} \otimes B^{\beta} \otimes A^{V \backslash \sigma \sqcup \beta}{ }_{\theta} \widetilde{H}^{*}\left(\operatorname{link}_{K_{V \backslash \beta}} \sigma ; \mathbb{k}\right) \otimes C^{\sigma} \otimes B^{V \backslash \sigma \sqcup \beta} \otimes A^{\beta}$, zдe $\sigma \in K, \beta \subset V$ u $\sigma \cap \beta=\varnothing$, и таким образом сохраняет доказанное в теореме 3 разложсение когомологий пространства $\mathscr{Z}_{K}\left(M^{n}, \partial M^{n}\right)$ в прямую сумму. зие.

Следующее предложение дает необходимое условие того, что $\mathscr{Z}_{K}\left(M^{n}, \partial M^{n}\right)$ - многообра-

ПреДЛОЖЕНИЕ 2. Если $\mathscr{Z}_{K}\left(M^{n}, \partial M^{n}\right)$ - многообразие, то $K$-горенштейнов ${ }^{*}$ комплекс.

Достаточным условием является то, что $K$ - симплициальная сфера.

Автор выражает глубокую благодарность своему научному руководителю профессору В.М. Бухштаберу за неоценимую помощь и внимание, проявленное на всех этапах написания данной статьи, а также доценту Т. Е. Панову за полезное обсуждение результатов настоящей работы.

\section{СПИСОК ЛИТЕРАТУРЫ}

[1] B. M. Бухштабер, Т. Е. Панов // УМН. 2000. T. 55. № 5. C. 3-106. [2] M. Davis, T. Januszkiewicz // Duke Math. J. 1991. V. 62. № 2. P. 417-451. [3] R. Stanley. Combinatorics and Commutative Algebra. Basel: Birkhäuser, 1996. [4] M. Hochster // Lecture Notes in Pure Appl. Math. 1977. V. 26. P. 171-223.

Московский государственньй университет им. М.В.Ломоносова E-mail: baskak@orc.ru
Принято редколлегией 04.09.2002 